\title{
A INTER-RELAÇÃO DAS SIALOMUCINAS (ANTÍGENOS TN E STN) COM 0 ADENOCARCINOMA NO ESÔFAGO DE BARRETT
}

\author{
Plínio Conte de faria Júnior*, Nelson Adami Andreollo, Miriam Aparecida da Silva Trevisan, luiz Roberto lopes \\ Trabalho realizado na Faculdade de Ciências Médicas da PUCCAMP e Departamento de Cirurgia e Gastrocentro da Faculdade de Ciências Médicas da \\ Unicamp - Campinas - SP
}

\author{
*Correspondência \\ Rua Álvaro Muller, 961 \\ Cep:130123-181, Campinas/SP. \\ Fone/Fax: (19) 3231-6190 \\ apmdefaria@uol.com.br
}

\begin{abstract}
RESUMO
OBJEтIvo. O esôfago de Barrett (EB) é consequêencia do refluxo gastroesofágico crônico e considerado fator de risco para o desenvolvimento de adenocarcinoma. Estudos do muco, em especial das mucinas ácidas representadas pelas sialomucinas presentes nas células caliciformes, mostraram que na metaplasia do tipo intestinal, o epitélio do órgão pode expressar antígenos denominados Tn e Stn. Estes antígenos já foram analisados em tumores gástricos e colônicos, porém não foram encontradas referências à sua utilização no EB. Este trabalho objetivou analisar estes antígenos em doentes com EB e em adenocarcinoma associado ao EB.

Métodos. Foram estudados, utilizando testes imunohistoquímicos, os antígenos Tn e Stn, nas biópsias endoscópicas de 29 doentes com EB, sete com adenocarcinoma no EB, além de oito indivíduos com epitélio esofágico normal.

Resultados. Nas células caliciformes, foi observada positividade para Stn em 100\% dos casos e para Tn em 48\% dos casos. Nas células colunares, o Stn foi sempre negativo, enquanto o Tn foi positivo em 100\% dos casos. Entretanto, nos doentes com adenocarcinoma no EB, a positividade para ambos os antígenos foi de 100\%. Nos indivíduos normais, houve positividade para 0 antígeno Tn e negatividade para Stn em todos os casos (100\%).

Conclusão. É provável que nos doentes com EB a positividade para o Tn, à semelhança do ocorrido quanto à positividade do mesmo antiggeno nos portadores de adenocarcinoma, possa significar maior suscetibilidade para ocorrência futura de câncer. Assim, a pesquisa das sialomucinas poderá ser rotineiramente utilizada, contribuindo como fator prognóstico para desenvolvimento de adenocarcinoma no EB.
\end{abstract}

Unitermos: Sialomucinas. Mucinas. Esôfago de Barrett. Adenocarcinoma.

\section{INTRODUÇÃO}

O esôfago de Barrett (EB) é o fator de risco mais importante para o desenvolvimento do adenocarcinoma de esôfago'. Estima-se que a incidência de adenocarcinoma seja de 30 a 125 vezes maior que na população geral, com ocorrência de $0,2 \%$ a $2,1 \%$ ao ano ${ }^{2-3}$.

A ocorrência de adenocarcinoma no EB está associada com a perda de alelos que incluem genes de supressão tumoral. Fortes evidências sugerem que 0 alvo da perda dos alelos do cromossomo $17 p$ é o gene de supressão tumoral p53, que se encontra ausente ou mutado em vários tumores do aparelho digestório. A identificação da proteína p53 no adenocarcinoma de esôfago varia de $53 \%$ a $87 \% \%^{4-5}$.

O estudo dos constituintes do muco do sistema digestório tem mostrado variações que poderiam ser utilizadas como fatores prognósticos em lesões consideradas como pré-neoplásicas no EB. As mucinas encontradas no muco são glicoproteínas de alto peso molecular, contendo mais de $85 \%$ de carboidratos, produzidas pelas células epiteliais do trato gastrointestinal, exercendo papel de proteção sobre a mucosa intestinal. Histoquimicamente são classificadas como ácidas ou neutras. As mucinas ácidas são subdivididas em sialomucinas e sulfomucinas, de acordo com a presença de ácido siálico ou grupos sulfato. As sialomucinas, por sua vez, são caracterizadas conforme a proporção existente de moléculas de $\mathrm{N}$-acetil ou de $\mathrm{O}$-acetil, derivadas do ácido siálico. As regiões produtoras de mucinas neutras são identificadas pela reação do PAS, enquanto o corante azul de Alcian identifica as ácidas, especialmente as sialomucinas, presentes nas células caliciformes (metaplásicas ou não) 6 .

No EB, o epitélio original do órgão passa a conter metaplasia intestinal e a expressar outros antígenos associados ao muco. Técnicas imunohistoquímicas permitiram um maior detalhamento do estudo do muco, a partir da deteç̧ão da expressão de oligossacarídeos, ou carboidratos, ligados às mucinas, tanto no epitélio original como no metaplásico. Assim, as sialomucinas Oacetiladas estão presentes nas metaplasias dos tipos I e llb, porém, próximo às células malignas, apenas sulfomucinas estavam presentes, estando ausentes as sialomucinas orto-acetiladas ${ }^{6}$. Achados semelhantes foram encontrados na mucosa adjacente aos adenocarcinomas de cólon, que mostraram a presença marcante de sialomucinas $\mathrm{N}$-acetiladas e redução tanto das sulfomucinas, quanto das sialomucinas $\mathrm{O}$-acetiladas. Além disso, nos carcinomas gástricos e na mucosa metaplásica adjacente (tipo IIb ou III) ficou demonstrada a presença de sulfomucinas e praticamente a ausência de mucinas $\mathrm{O}$-acetiladas ${ }^{7-9}$. 
Lapertosa et al. $(\text { 1992 })^{10}$ demonstraram que o padrão secretório das mucinas no epitélio displásico/carcinomatoso que se desenvolve no EB é similar ao encontrado no carcinoma gástrico, sugerindo que a diminuição e mesmo a ausência de enzimas $\mathrm{O}$ acetiladas, sugere imaturidade celular.

Chejfec et al. (1992)6 afirmam que, independentemente do subtipo da metaplasia encontrada no Barrett, as sulfomucinas são encontradas na maioria dos casos, bem como nas neoplasias associadas. $\mathrm{Na}$ transição esôfago-gástrica (TEG), as alterações observadas são diferentes das encontradas nos demais locais do organismo. Aliás, o próprio epitélio expressa positividade para citoqueratina de forma diferente dos demais epitélios do trato gastrointestinal ${ }^{10}$.

As mucinas sendo glicoproteínas são compostas basicamente por uma cadeia protéica à qual cadeias laterais de oligossacarídeos heterogêneos estão unidos por ligações covalentes. Cada uma dessas cadeias laterais é conectada a resíduos de serina ou treonina pelo açúcar GalNAc. Tal processo, conhecido como glicosilação, determina a formação do chamado antígeno Tn. Uma vez sintetizado, o antígeno Tn pode ser novamente glicosilado pela adição de açúcares específicos, sob a ação da enzima glicosiltransferase. A adição do ácido siálico forma o antígeno sialosyl-Tn (Stn), produto final que não sofre nova glicosilação. Ambos os antígenos são considerados "antígenos associados a tumor" e seu acúmulo identificaria a glicosilação aberrante, um fenômeno associado ao câncer ${ }^{7-9,11-13}$.

O objetivo do presente estudo foi analisar as sialomucinas (antígenos Tn e Stn) em doentes com EB e com adenocarcinoma associado ao $E B$, uma vez que não foram encontradas referências na literatura sobre este assunto.

\section{Métodos}

Foram estudados 44 indivíduos em seguimento nos ambulatórios de dois hospitais universitários (Hospital de Clínicas da Unicamp ou Hospital e Maternidade Celso Pierro da PUCCAMP), divididos em três grupos, segundo critérios clínicos, endoscópicos e histopatológicos apresentados a seguir:

Grupo I - Doentes com EB $(n=29)$ : 20 pacientes do sexo masculino e nove do sexo feminino, com média de idade de 56, I anos, variando entre 37 e 70 anos, submetidos a exame endoscópico devido a sintomas da doença do refluxo gastroesofágico (DRGE) como queimação retroesternal e regurgitação de secreção ácida por período prolongado. Apresentavam ao exame endoscópico a mucosa com características de EB, com extensão de pelo menos $2 \mathrm{~cm}$., não necessariamente circunferencial. Em todos os casos, foram realizadas biópsias das áreas suspeitas.

Grupo II - Doentes com adenocarcinoma no epitélio de Barrett $(n=7)$ : sete doentes, todos do sexo masculino, com média de idade de 65,3 anos, variando entre 54 e 76 anos, submetidos ao exame endoscópico por sintomas relacionados à DRGE, sendo que três apresentavam sintomas como disfagia, odinofagia e emagrecimento significativo, com perda ponderal de aproximadamente dez quilos nos três meses anteriores.
Desse grupo, cinco doentes tinham EB comprovado histologicamente. Os outros dois doentes tiveram o diagnóstico de câncer feito no primeiro exame endoscópico, sendo que o endoscopista verificou que a mucosa adjacente à lesão neoplásica apresentava características de EB, tendo sido esta observação confirmada por meio de biópsias.

Grupo III - Indivíduos normais $(n=8)$ (controles): sendo cinco do sexo masculino e três do sexo feminino, com média de idade de 37,2 anos, variando entre 27 e 38 anos, selecionados entre os submetidos a endoscopia digestiva alta por sintomas dispépticos não relacionados à DRGE, com esôfago distal de aspecto normal à endoscopia, e cujas biópsias não evidenciavam metaplasia intestinal.

\section{Padronização para obtenção das biópsias endoscópicas}

Apesar da imagem endoscópica de coloração salmão ser suficiente na grande maioria das vezes para que houvesse a suspeita de $E B$, em todos os doentes foi realizada cromoscopia utilizando o lugol, que cora somente as células da mucosa esofágica (epitélio escamoso) ricas em grânulos de glicogênio, ou o azul de metileno, corante que é absorvido pelo epitélio metaplásico, fazendo com que o mesmo adquira uma coloração azul de forte intensidade. Em seguida, foram realizadas biópsias em número não inferior a seis, nos quatro quadrantes do órgão, a partir da margem mais distal do suposto epitélio metaplásico, a intervalos de $2 \mathrm{~cm}$, quando a extensão do mesmo assim o permitia. Quando o epitélio metaplásico não possuía extensão suficiente, eram retirados pelo menos quatro fragmentos.

\section{Estudo histopatológico}

Os cortes histológicos obtidos nas biópsias foram corados com hematoxilina-eosina (HE) e avaliados no Departamento de Anatomia Patológica da FCM-UNICAMP. Todas as lâminas também foram coradas com o azul de Alcian, em pH 2,5 para confirmação da presença de células caliciformes e conseqüentemente do EB.

\section{Estudo imunohistoquímico}

Os estudos dos antigenos Th e Stn foram realizados no Laboratório de Patologia Experimental da Unicamp. A reação para imunooperoxidase foi realizada seguindo os padrões definidos previamente e as lâminas dos estudos histopatológicos e imunohistoquímicos foram revisadas e analisadas pelo mesmo anátomo-patologista.

\section{Resultados}

\section{Doentes com esôfago de Barrett}

Nos 29 doentes com EB, foi observada diferença na expressão das mucinas das células caliciformes. Em todos houve positividade para Stn e em 14 houve positividade concomitante para Tn. As células colunares foram muito constantes nas reações e com expressão exatamente igual a das células colunares da TEG, isto é, todas positivas para $\mathrm{Tn}$ e negativas para Stn (Tabela I). 
Tabela I - Positividade dos antígenos Stn e Tn nas células caliciformes e colunares, nos portadores de EB (29 doentes)

\begin{tabular}{|c|c|c|}
\hline & Células caliciformes & Células colunares \\
\hline $\begin{array}{l}\text { Stn } \\
\text { Tn }\end{array}$ & $\begin{array}{l}29 / 29-(100 \%) \\
14 / 29-(48,2 \%)\end{array}$ & $\begin{array}{c}0 / 29-(0 \%) \\
29 / 29-(100 \%)\end{array}$ \\
\hline
\end{tabular}

A reação do PAS nas células colunares dos doentes com EB demonstrou a presença de mucinas neutras em 17 casos, com distribuição semelhante a do tipo II da metaplasia intestinal, segundo JASS et al. (1980). Em quatro casos foi semelhante ao foveolar gástrico e, em nove, o padrão foi misto, com bordadura em escova, epitélio tipo foveolar e metaplasia intestinal tipo II.

\section{Esôfago de Barrett e adenocarcinoma}

Os sete casos de adenocarcinoma originados no EB mostraram 100\% de positividade citoplasmática difusa e de membrana ao Stn e ao Tn. Um deles mostrou uma porção superficial vilosa mais diferenciada, na qual a reação para Stn foi negativa, porém na profundidade, a positividade foi nítida. Além disso, em quatro dos sete casos, o estudo da mucosa não neoplásica adjacente mostrou positividade concomitante para Stn e Tn nas células caliciformes em dois casos.

\section{Indivíduos normais}

Nos oito indivíduos com TEG normal (controles), as células colunares foram consistentemente negativas para Stn e positivas para $T n$, sendo que nestas últimas foi verificada a presença de grânulos supranucleares.

\section{Discussão}

\section{Esôfago de Barrett e adenocarcinoma}

Vários estudos demonstram que a frequêencia de adenocarcinoma na transição esôfago-gástrica tem aumentado nos Estados Unidos nas últimas duas décadas, mais que qualquer outro tipo de câncer. O risco de degeneração maligna, estimado em cerca de 30 a 125 vezes maior do que na população geral, parece estar relacionado à maior extensão do epitélio metaplásico, não significando, porém, que os doentes com segmentos curtos de EB também não estejam sob risco de desenvolverem neoplasia ${ }^{2-3,14-16}$.

A incidência real de adenocarcinoma em doentes com EB é difícil de ser determinada, embora estudos retrospectivos registram que variou de 1:55 a 1:441 (Tabela 2), enquanto em estudos prospectivos, variou de 1:52 a 1:208 (Tabela 3). Houve grande variação dos valores por ano de seguimento nos estudos retrospectivos (Tabela 2), justificadas por diversas razões, relacionadas a seguir:

a) Os estudos incluíram doentes que tinham somente epitélio ou de tipo fúndico ou, cárdico, ou seja, que não teriam risco aumentado de desenvolverem adenocarcinoma;

b) A maioria corresponde a estudos retrospectivos;

c) Os trabalhos relacionados foram realizados em serviços de referência para o estudo de adenocarcinoma se desenvolvendo no $E B$, com maior probabilidade de receberem doentes com suspeita de displasia do que em hospitais/serviços gerais.

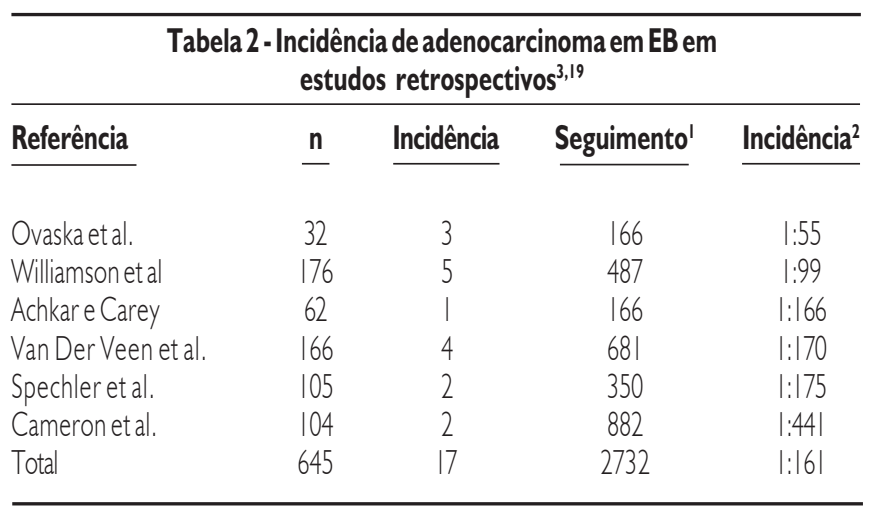

Em meses

${ }^{2} \mathrm{Em}$ anos de seguimento

\begin{tabular}{|c|c|c|c|c|c|}
\hline \multicolumn{6}{|c|}{$\begin{array}{l}\text { Tabela } 3 \text { - Incidência de metaplasia intestinal associada ao } \\
\text { adenocarcinoma em EB em estudos prospectivos }\end{array}$} \\
\hline Referência & $\mathrm{n}$ & $\begin{array}{c}\text { Metaplasia } \\
\text { intestinal }\end{array}$ & $\underline{\text { Incidência }}$ & Seguimento' & Incidência ${ }^{2}$ \\
\hline $\begin{array}{l}\text { Hammeeteman etal. } \\
\text { Bonelli etal. } \\
\text { Robertson etal. } \\
\text { Miros etal. } \\
\text { Iftikhar et al. } \\
\text { Drewitz etal. } \\
\text { Total }\end{array}$ & $\begin{array}{l}50 \\
71 \\
56 \\
81 \\
102 \\
170 \\
530\end{array}$ & $\begin{array}{l}68 \\
100 \\
89 \\
78 \\
N D \\
100\end{array}$ & $\begin{array}{c}5 \\
2 \\
4 \\
3 \\
4 \\
4 \\
21\end{array}$ & $\begin{array}{l}260 \\
110 \\
224 \\
289 \\
462 \\
834 \\
2179\end{array}$ & $\begin{array}{l}1: 52 \\
1: 55 \\
1: 56 \\
1: 96 \\
\mid: 115 \\
1: 208 \\
\mid: 104\end{array}$ \\
\hline
\end{tabular}

Em meses

${ }^{2} \mathrm{Em}$ anos de seguimento

Sendo assim, para a obtenção de dados mais fiéis com relação à incidência de adenocarcinoma associado ao EB, é mais adequada a realização de estudos prospectivos em um número maior de doentes com biópsias endoscópicas do epitélio colunar especializado, que por ocasião da inclusão para análise ainda não sejam portadores de adenocarcinoma de esôfago ${ }^{3,17}$.

É de grande importância estabelecer a real incidência de câncer no EB, também para que se possa definir o tipo de seguimento a ser adotado, uma vez que os custos envolvidos são elevados, havendo uma estimativa de $\cup \$ 62.000$ e perda de 78 dias de trabalho por ano para cada caso de câncer diagnosticado, segundo o estudo de Achkar e Carey ${ }^{18}$, revisto por Seabrook et al..$^{19}$.

Dados epidemiológicos sugerem que, a despeito do aumento da incidência do adenocarcinoma de esôfago, a maioria dos indivíduos com EB não desenvolverão neoplasia. Entretanto, os atuais protocolos de seguimento recomendam um exame endoscópico a cada dois anos, realizando biópsias da mucosa com aspecto metaplásico à macroscopia nos quatro quadrantes do órgão a cada $2 \mathrm{~cm}$ para os pacientes sem evidência histológica de displasia e a cada 6 a 12 meses para pacientes com displasia de baixo grau. Nos pacientes com displasia de alto grau, o diagnóstico sendo confirmado por mais de um patologista experiente, está indicada a ressecção cirúrgica do órgão. Entretanto, outros autores recomendam a 
realização de exame endoscópico com biópsias a cada três meses, na tentativa de diagnosticar uma eventual neoplasia o mais precocemente possível ${ }^{2,20}$.

\section{Estudo do muco}

Umpleby et al. $(1985)^{21}$ demonstraram que uma das características importantes dos tumores de cólon seria a sua capacidade de produção de muco, que estaria relacionada com o comportamento biológico da neoplasia. E durante muitos anos os carcinomas mucinosos, que representam de 15 a $20 \%$ dos carcinomas coloretais, foram considerados os de pior prognóstico, apresentando taxas elevadas de recorrência e baixas de sobrevida, principalmente quando o componente mucinoso do tumor ocupava mais de $60 \%$ do seu volume. Entretanto, Halvorsen e Seim ${ }^{22}$ não observaram diferenças estatísticas no seguimento dos tumores colo-retais mucinosos e não-mucinosos, demonstrando que o grupo controle composto de carcinomas "não-mucinosos" era constituído de adenocarcinomas, muitos dos quais produtores de muco.

Portanto, seria pouco confiável basear-se somente na quantidade de muco produzido pelo tumor, para que avaliações quanto ao seguimento clínico pudessem ser feitas, uma vez que diferenças importantes quanto ao prognóstico poderiam estar relacionadas a mudanças na qualidade do muco produzido pelo mesmo. Itzkowitz et al. ${ }^{12}$, com o objetivo de investigar esta possibilidade, estudaram as relações entre o prognóstico do câncer colo-retal e a expressão do antígeno sialosyl-Tn (Stn), utilizando anticorpos monoclonais, por meio de reações imunohistoquímicas. Além do antígeno Stn, analisaram também a expressão de outros dois antígenos, denominados $T n$ e $T$, demonstrando que ambos são altamente específicos e sensíveis em tumores do cólon. O Stn não é expresso na mucosa colo-retal normal, mas é demonstrado na maioria dos cânceres primários que venham a se desenvolver nessa mucosa. É uma variável que não depende do sexo, idade, ploidia do tumor, localização, estádio de Dukes, profundidade da invasão, ou ainda do tipo histológico, estando presente sempre nos casos de pior evolução, além de ser útil no diagnóstico, pois foi verificado em quase $80 \%$ dos casos. Relatam, também, que a associação da expressão com o pior prognóstico depende da presença do ácido siálico juntamente com o antígeno Stn.

Os resultados obtidos por Itzkowitz et al. ${ }^{12}$ indicam que a expressão de Stn pelas células do câncer colo-retal está fortemente associada com o prognóstico e que o grupo de doentes que não expressaram o antígeno tiveram 100\% de sobrevida em cinco anos, enquanto os doentes Stn positivos somente $73 \%$ de sobrevida.

Também David et al. ${ }^{13}$ estudaram a expressão dos antígenos Tn, Stn e T, através de estudos imunohistoquímicos, utilizando anticorpos monoclonais específicos na mucosa de aspecto normal adjacente aos carcinomas gástricos $(n=58)$, nos tumores primários $(n=87$ ), e nas metástases (linfonodais (329) e hepáticas(2)). Observaram que na mucosa de aspecto normal ocorreu expressão de Tn em todos os casos, de Stn em oito casos, não tendo ocorrido expressão do antígeno T. Na metaplasia intestinal da mucosa gástrica, as células caliciformes foram positivas em sua totalidade para o Stn e as células colunares apresentaram positividade granular supranuclear para o Tn, e também para o Stn, na metade dos casos. Nas neoplasias, ambos passam a ter positividade difusa no citoplasma.

Na presente casuística de EB, as células colunares foram positivas para o Tn e negativas para o Stn nos 29 casos, semelhante ao epitélio normal da transição esôfago- gástrica e, como foi demonstrado em células colunares de diversos epitélios, segundo dados da literatura. Por outro lado, as células caliciformes foram positivas para o Stn nos 29 casos, e para o Tn, em 14.

Como o Tn é encontrado em adenocarcinomas produtores de muco, deve-se procurar interpretar o motivo da presença de células caliciformes $\mathrm{Tn}$ positivas. Elas poderiam representar uma transformação da célula colunar em célula caliciforme, com a manutenção dos antígenos originais da célula colunar. Esta hipótese estaria de acordo com as explicações de Duchatelle et al." , que referem a existência de uma possível "mucosa cárdica modificada", constituída por células "ball shaped", que contém muco positivo para PAS-AB, sulfomucina e anti-LIMA "large intestin mucus antigen", e que seria um epitélio intermediário entre a mucosa cárdica normal e a com metaplasia intestinal.

Por outro lado, Jass et al. ${ }^{8}$ demonstraram a presença de Tn em células caliciformes do cólon após a utilização da neuroaminidase, para desmascaramento do antígeno. Nesta pesquisa, esse procedimento não foi realizado e por isso considerou-se a possibilidade de ter-se detectado a expressão de outra mucina com sua produção desregulada (super- regulada). Outra hipótese é a de que a mucina habitual possa ter perdido parte da cadeia de oligossacarídeos no processo de regeneração da mucosa, tornando-se novamente positiva para $\circ \mathrm{Tn}$.

De qualquer forma, células caliciformes positivas ao mesmo tempo para Tn e Stn foram encontradas em metade dos doentes da presente casuística, e podem estar sinalizando os casos que efetivamente necessitam seguimento.

$\mathrm{Na}$ literatura consultada notou-se diferentes interpretações dos autores em relação à positividade desses anticorpos em mucosa normal, e com alterações não-neoplásicas, como a metaplasia intestinal. Analisando as publicações referentes à esta questão, o que foi observado é que não são utilizados os mesmos anticorpos monoclonais. Portanto, os dados não podem ser pareados. Também as correlações com reações histoquímicas são restritas, cada qual abordando apenas um pequeno segmento do problema $a^{7-9,12-13}$.

Recentes publicações de Chaves et al. ${ }^{23}$ e Amano et al. ${ }^{24}$ estudando doentes com epitélio colunar no esôfago e adenocarcinoma, constataram que a expressão da mucina e a presença do fenótipo positivo para mucina na metaplasia intestinal associada, poderão ser úteis na estratificação do risco de neoplasia no esôfago de Barrett.

Portanto, para se entender o que realmente ocorre no EB seria necessário um estudo bem mais amplo do muco e de outras alterações celulares próprias da transição esôfago-gástrica, possibilitando uma visão global do processo. 
FARIA JÚNIOR PC ET AL.

\section{ConClusão}

Tendo em vista os conhecimentos atuais, o grupo de 14 doentes com positividade para $T n$ merece seguimento a médio e a longo prazo, realizando endoscopia digestiva alta e biópsias do EB, pois seriam os com maior possibilidade de ocorrência de neoplasia. Em um futuro próximo, a pesquisa das sialomucinas poderá ser rotineiramente utilizada, desde que outros estudos com maior número de doentes confirmem sua importância como fator prognóstico para desenvolvimento de adenocarcinoma no EB.

Conflito de interesse: não há.

\section{SUMMARY}

\section{Relationship of the sialomucins (TN and Stn antigens) With ADENOCARCINOMA IN BARRETT'S ESOPHAGUS}

OBJECIVE. Barrett's esophagus (BE) is a consequence of chronic gastroesophageal reflux and is considered a risk factor for adenocarcinoma. The study of the mucus, especially acid mucins, such as the sialomucins in the goblet cells which characterize BE, showed that in intestinal metaplasia, frequent in the digestive tract, the organ's original epithelium could express $\mathrm{Tn}$ and Stn antigens. These antigens have already been detected in gastric and colonic tumors, however references in BE were not found. This research aimed to analyze these antigens in patients with BE and in adenocarcinoma associated with $B E$.

Methods. Utilizing immunohistochemistry tests, Tn and Stn antigens were studied in the endoscopic biopsies of 29 patients with $B E$ and seven with adenocarcinoma in $B E$, as well as eight individuals with normal esophageal epithelium at upper digestive endoscopy..

RESULTS. The Stn antigen was positive in the goblet cells of patients with BE in 100\% of the cases and the Tn was positive in $48 \%$. In the columnar cells, Stn was always negative, while Tn was positive in 100\% of the cases. However, in adenocarcinoma in BE, both antigens were $100 \%$ positive. In normal individuals, the $\mathrm{Tn}$ antigen was positive and the antigen Stn negative in all cases.

CONCLUSION. It is probable that the BE group in which the $T n$ antigens in the goblet cells are positive, similarly to the same antigen in the adenocarcinoma group, might indicate a higher susceptibility for potential occurrence of cancer. In the future, trials with sialomucins could be used routinely, thereby contributing as a prognostic factor of adenocarcinoma in BE. [Rev Assoc Med Bras 2007; 53(4): 360-4]

KEY wORDS: Sialomucin. Mucin. Barrett's Esophagus. Adenocarcinoma.

\section{REFERÊNCIAS}

I. Menke-Pluymers MbE. Risk factors for neoplastic progression in Barrett's mucosa. Eur J Surg Oncol. 1996;22:31 I-6.

2. Falk GW. Barrett's esophagus. Gastrointest Endosc Clin Noth Am. 1994; 4: 773-89.

3. Drewitz DJ, Sampliner RE, Garewal HS. The incidence of adenocarcinoma in Barrett's esophagus: a prospective study of 170 patients followed 4,8 years. Am J Gastroenterol. 1997;92:212-5.

4. Flejou JF, Potet F, Muzeau F, Le Pelletier F, Fekete F, Henin D.
Overexpression of p53 protein in Barrett's syndrome with malignant transformation. J Clin Pathol. 1993;46:330-3.

5. Ramel S, Reid, BJ, Sanchez CA. Evaluations of p53 protein expression in Barrett's esophagus by two-parameter flow cytometry. Gastroenterology. 1992;102:1220-8.

6. Chejfec, G, Schnell T, Sontag S. Barrett's esophagus: a preneoplastic disorder. Am J Clin Pathol. 1992;98:5-7.

7. Peuchmaur M, Potet F, Goldfain D. Mucin histochemistry of the columnar epithelium of the oesophagus (Barrett's oesophagus): a prospective biopsy study. J Clin Pathol. 1984;37:607-I0.

8. Jass JR, Allison LJ, Edgar SG. Distribution of sialosyl Tn and Tn antigens within normal and malignant colorectal epithelium. J Pathol. 1995; 176:143-9.

9. Jass JR. Role of intestinal metaplasia in the histogenesis of gastric carcinoma. J Clin Pathol. 1980;33:801-10.

I0. Lapertosa G, Baracchini P, Fulcheri E. Mucin histochemical analysis in the interpretation of Barrett's esophagus. Results of a multicenter study. The Operative Group for the Study of Esophageal Precancer. Am J Clin Pathol. 1992;98:61-6.

II. Duchatelle V, Potet F, Bara J, MA J, Goldfain D. Mucin immunohistochemistry of the columnar epithelium of the oesophagus (Barrett's oesophagus). Virch Arch A Pathol Anat. 1989:4|4:359-63.

12. Itzkowitz SH, Blomm EJ, Kokal WA Modin G, Hakomori S, Kim YS. Sialosyl-Tn: a novel mucin antigen associated with prognosis in colorectal cancer patients. Cancer Res. 1990;66:1960-6.

13. David L, Nesland JM, Clausen H, Carneiro F, Sobrinho-Simões M. Simple mucin-type carbohydrate antigens ( $T n$, Sialosyl-Tn and T) in gastric mucosa, carcinoma and metastases. APMIS 1992;27(Suppl): I 62-72.

14. Blot WJ, Devesa SS, Kneller RW. Rising incidence of adenocarcinoma of the esophagus and gastric cardia. JAMA. 1991;265:1287.

15. Cameron AJ, Lombay CT. Barrett's esophagus: age prevalence, and extent of columnar epithelium. Gastroenterology. 1992; 103:1241-5.

16. Andreollo NA, Michelino UM, Brandalise NA, Lopes LR, Trevisan MAS, Leonardi LS. Incidência e Epidemiologia do Epitélio de Barrett no Gastrocentro - Unicamp. Arq Gastroenterol. 1997;34:22-6.

17. Reid BJ. Barrett's esophagus and esophageal adenocarcinoma. Gastroenterol Clin North Am. 1991;20:817-34.

18. Achkar E, Carey, W. The cost of surveillance for adenocarcinoma complicating Barrett's esophagus. Am J Gastroenterol. 1988;83:291 4.

19. Seabrook M, Holt S, Gilrane T. Barrett's esophagus: observations on diagnosis and mangement. South Med J. 1992;85:280-8.

20. Sampliner RE. Practice guidelines on the diagnosis, surveillance, and therapy of Barrett's esophagus. Am J Gastroenterol. 1998;93:102832.

21. Umpleby HC, Ranson DI, Willians RCN. Peculiarities of mucinous colorectal carcinoma. Br J Surg. 1985;72:715-8.

22. Halvorsen TB, Seim E. Influence of mucinous components on survival in colorectal adenocarcinomas: a multivariate analysis. J Clin Pathol. 1988;41:1068-72

23. Chaves P, Cruz C, Dias Pereira A, Suspiro A, De Almeida JC, Leitao $\mathrm{CN}$, et al. Gastric and intestinal differentiation in Barrett's metaplasia and associated adenocarcinoma. Dis Esophagus. 2005; | 8:383-7.

24. Amano Y, Kushiyama Y, Yuki T, Takahashi Y, Moriyama I, Fukuhara $\mathrm{H}$, et al. Prevalence of and risk factors for Barrett's esophagus with intestinal predominant mucin phenotype. Scand J Gastroenterol. 2006;41:873-9. 